\title{
Fatores que Afetam o Comportamento Ambiental de Residentes em Destinos Turísticos Costeiros
}

\author{
Amanda Mirely Cipriano Soares \\ Sérgio Marques Júnior ${ }^{b}$ \\ Márcio Marreiro das Chagas ${ }^{c}$
}

\section{Resumo}

Considerada um relevante local de visitação, a zona costeira brasileira é um dos espaços mais procurados por turistas nacionais einternacionais. Entretanto, observa-se significativo impacto ambiental gerado pela atividade humana, principalmente no que se refere à geração de resíduos sólidos, poluição das águas e construções indevidas, entre outros. Nesse sentido, o objetivo deste estudo foi investigar as inter-relações entre fatores capazes de influenciar o comportamento ambiental de residentes em destinos turísticos costeiros. Para tanto, propôs-se um estudo quantitativo, utilizando o método hipotético-dedutivo, uma vez que são utilizados modelos teóricos para propor hipóteses. 0 estudo teve como objeto os residentes do município de Baía Formosa, destino turístico em consolidação, localizado no litoral sul do estado do Rio Grande do Norte, Brasil, e utilizou uma amostragem do tipo não probabilística, que resultou na aplicação de 288 questionários. 0 instrumento de coleta de dados foi um questionário composto por dimensões baseadas na teoria do comportamento planejado, sendo adicionado o constructo "apego ao lugar". As variáveis que compuseram essas dimensões foram avaliadas por meio de uma escala métrica de onze pontos (baseada na escala Likert), a qual variou entre 0 (Discordo plenamente) a 10 (Concordo plenamente), conforme o nível de concordância com uma frase proposta para caracterizar cada variável. Os dados coletados foram analisados pelo método da modelagem de equações estruturais. Os resultados apontam que as variáveis de caráter cognitivo têm maior poder explicativo do comportamento ambiental de residentes no destino analisado do que as de caráter afetivo.

Palavras-chave: Comportamento ambiental de residentes; Destinos turísticos costeiros; Gestão ambiental.

\section{Abstract \\ Factors Affecting Residents Environmental Behavior in Coastal Tourism Destinations}

Brazilian coastal zone is considered a relevant place of visitation, one of the most desirable places for national and international tourists. However, there is significant environmental impact generated by the activity, mainly regarding solid waste generation, water pollution and undue construction, among others. In this sense, this study aimed

a. Mestre em Turismo pelo Programa de Pós-Graduação em Turismo da Universidade Federal do Rio Grande do Norte (PPGTUR/UFRN), Natal, Rio Grande do Norte, Brasil. E-mail: amandamirellybf19@gmail.com

b. Doutor em Agronomia pela Universidade Estadual Paulista (Unesp), Botucatu, São Paulo, Brasil. Professor do PPGTUR/UFRN. E-mail: sergio@ct.ufrn.br

c. Doutor em Administração pela UFRN. Professor do curso superior de Tecnologia em Gestão de Turismo do Instituto Federal de Educação, Ciência e Tecnologia do Rio Grande do Norte (IFRN), Canguaretama, RN, Brasil. E-mail: marcio_marreiro@yahoo.com.br 
to investigate the dependence relations between antecedents capable of influencing residents environmental behavior in coastal tourist destinations. For that, a quantitative study was proposed, using hypothetical-deductive method, since some theoretical models are used to propose some hypotheses. The study object was residents of Baía Formosa, a tourist destination in consolidation process, located at Rio Grande do Norte state, Brazil, using a non-probalistic sampling, which resulted in the application of 288 questionnaires. The data collection instrument was composed by dimensions and variables based on Theory of Planned Behavior and Place Attachment construct. The variables composing these dimensions were evaluated using an eleven-point metric scale (based on the Likert scale), which ranged from 0 (I strongly disagree) to 10 (I fully agree), according to the level of agreement with a proposed sentence to characterize each tested variable. The collected data were analyzed by Structural Equation Modeling method. Results show that the cognitive variables have a greater explanatory power of residents environmental behavior than affective variables.

Keywords: Residents environmental behavior; Coastal tourist destinations; Environmental management.

\section{Resumen}

\section{Factores que afectan el comportamiento ambiental de residentes en destinos turísticos costeros}

La zona costera brasileña es considerada un relevante local de visitación, uno de los espacios más buscados por turistas nacionales e internacionales. Sin embargo, se observa un significativo impacto ambiental generado por la actividad, principalmente en lo que se refiere a la generación de residuos sólidos, contaminación de las aguas y construcciones indebidas, entre otros. En este sentido, el objetivo de este estudio fue el de investigar las interrelaciones entre factores capaces de influenciar el comportamiento ambiental de residentes en destinos turísticos costeros. Para ello, se propuso un estudio cuantitativo, utilizando el método hipotético-deductivo, ya que se utilizan algunos modelos teóricos para proponer algunas hipótesis. El estudio tuvo como objeto los residentes del municipio de Baía Formosa, destino turístico en consolidación, ubicado en el litoral sur del Estado de Rio Grande do Norte, Brasil, utilizando un muestreo del tipo no probalístico, que resultó en la aplicación de 288 cuestionarios. El instrumento de recolección de datos fue un cuestionario compuesto por dimensiones basadas en la Teoría del Comportamiento Planeado, agregando el constructo Apego al Lugar. Las variables que compusieron estas dimensiones fueron evaluadas por medio de una escala métrica de once puntos (basada en la escala Likert), la cual varía entre 0 (desacuerdo plenamente) a 10 (estoy totalmente de acuerdo), según el nivel de concordancia con una frase propuesta para caracterizar cada variable. Los datos recolectados fueron analizados por el método del modelado de ecuaciones estructurales. Los resultados apuntan que las variables de carácter cognitivo tienen un mayor poder explicativo del comportamiento ambiental de residentes en el destino analizado que las variables de carácter afectivo.

Palabras clave: Comportamiento ambiental de residentes; destinos turísticos costeros; Gestión ambiental.

\section{INTRODUÇÃo}

As sociedades globais vêm passando por um intenso processo de transformação decorrente, principalmente, de um sistema econômico que busca o aumento da competitividade das organizações. Segundo a The Worldwatch Institute (2013), entre 1992 e 2012, estima-se que houve um aumento de 1,6 
bilhão de pessoas no mundo, implicando o acréscimo de 50 trilhões de dólares do PIB global. Esse crescimento foi gradativo, gerou um maior consumo de bens e serviços e, consequentemente, crescente utilização dos recursos naturais.

Além do fator demográfico, verifica-se o desenvolvimento econômico de algumas atividades, como o turismo, destacando-se pelo grande potencial de fomentar a economia de um país que - no caso do Brasil, representa aproximadamente 3,7\% do PIB nacional (Ministério do Turismo, 2016). Observa-se que o crescimento do turismo vem se tornando significativo em espaços naturais porque as pessoas estão se direcionado para os "ambientes verdes" na tentativa de se refugiarem de problemas urbanos, como estresse, violência e reposição de condições físicas e mentais (Carvalho, 2016).

No entanto, tal fenômeno tem contribuído para o crescimento acelerado do processo de urbanização, gerando, em certos casos, a degradação dos espaços, principalmente a devastação da fauna e flora (Le, Hollenhorst, Harris, McLaughlin, \& Shook, 2006; Fandé \& Pereira, 2014). A zona costeira brasileira também se tornou palco de exploração desse público, constituindo-se em um dos espaços mais afetados pela ação antrópica. De imediato, observa-se a relevância ambiental desses limites costeiros, já que abarcam uma área de $8.698 \mathrm{~km}^{2}$ de extensão, compreendendo um espaço de aproximadamente $388.000 \mathrm{Km}^{2}$ de ecossistemas e 44 milhões de habitantes (Oliveira \& Nicolodi, 2012).

De fato, problemas ambientais não podem ser associados apenas ao acelerado crescimento da urbanização, mas também à conduta ou comportamento humano (Maloney \& Ward, 1973), o que vem fomentando a elaboração de estudos que visam compreender até que ponto a ação humana pode afetar o meio ambiente. Em termos de gestão urbana, para a obtenção de melhores resultados, faz-se necessário definir um tipo de comportamento orientado à manutenção dos recursos naturais, chegando-se a ideia de comportamento ambiental, como pode ser observado em Bonnes e Bonaiuto (2002), Corral-Verdugo e Pinheiro (1999) ou comportamento pró-ambiental, como em Karp (1996).

Tendo esta proposição como base, o objetivo do presente estudo foi o de investigar a inter-relação entre fatores capazes de influenciar o comportamento ambiental de residentes em destinos turísticos costeiros.

\section{MARCO TEÓRICO}

\section{Impactos ambientais em destinos turísticos costeiros}

Ao longo do tempo, o processo de desenvolvimento turístico foi se consolidando em muitos territórios, sustentado no pensamento antropocêntrico e economicista da natureza como fonte inesgotável de recursos (Le et al., 2006). Mesmo que se tenha observado indicadores econômicos positivos, impactos negativos foram gerados no meio ambiente natural, sobretudo em áreas onde os principais atrativos se encontrem nas zonas costeiras. Entre os impactos negativos provocados pelo turismo em regiões costeiras, destaca-se a instalação da infraestrutura, assim como a falta dela, no caso da geração, recolhimento e disposição adequada de resíduos sólidos e de esgotos (Vasconcelos \& Coriolano, 2008). 
Impactos ambientais em destinos costeiros são ressaltados em estudos como os de Smith (1992), Duim e Calders (2002), Moura-Fé e Pinheiro (2013), Fandé e Pereira (2014). Esses trabalhos, além de identificar as causas do impacto negativo (aumento de resíduos sólidos, indústria petrolífera, expansão do turismo e crescimento imobiliário), colocam também em discussão a efetividade de algumas alternativas consideradas "sustentáveis", geralmente implantadas nessas áreas com o intuito de sanar problemas ambientais, afetando a vida da população receptora. Algumas ações têm sido propostas para minimizar os impactos ambientais da atividade, como criação de áreas de proteção ambiental, aprofundamento nos estudos sobre técnicas de prevenção e conservação de áreas naturais, fiscalização de atividades potencialmente poluidoras, criação de zoneamento ecológico-econômico, programas de educação e monitoramento ambiental, entre outras.

Tais instrumentos corroboram medidas mais proativas e induzem resultados mais positivos para organizações, uma vez que a gestão ambiental trata-se de um processo de aplicação de inovação para alcançar a sustentabilidade, responsabilidade social e criação de vantagem competitiva por meio de aprendizagem contínua e desenvolvimento, abraçando metas e estratégias ambientais integradas com os objetivos e estratégias da organização (Haden, Oyler, \& Humphreys, 2009, p. 11).

Keene e Pullin (2011) argumentam que a efetividade de programas e políticas ambientais vem à tona porque muitos não são testados e, ainda, são obstruídos por barreiras técnicas, culturais e políticas. Logo, o caminho para se alcançar a eficácia e tornar práticas ambientais mais diligentes, apesar de ser um processo lento, é, além de fluxo de informações e auto-organização, a cooperação e integração das partes envolvidas no processo.

Observa-se que implantar ações de gestão ambiental em um destino turístico costeiro é complexo, pois envolve inúmeras visões e estratégias, podendo abarcar objetivos diferenciados, variando de acordo com as concepções de cada gestor. No tocante ao comportamento humano, entende-se que a gestão ambiental, como instrumento de valoração dos interesses ambientais, é eficaz, no sentido de atrair pessoas com condutas éticas para uma destinação, bem como para estimular o comportamento pró-ambiental de residentes.

Para Pol (2003), o estudo do comportamento humano é fundamental para a efetivação da gestão ambiental, uma vez que para a elaboração de projetos há a necessidade de se avaliar a aceitação social do processo. Além do mais, os procedimentos, estratégias e normas utilizadas pela gestão ambiental estão pautadas na mudança de valores e atitudes dos indivíduos em relação ao meio natural. 0 autor ressalta que a produção de um produto ecologicamente mais correto pode ser resumida em um ciclo que envolve tendências dos clientes potenciais e reais, como também engloba possíveis comportamentos que podem interferir nesse processo.

Também é parte importante levar em consideração as diferenças étnicas e culturais, dado que, por exemplo, povos indígenas exercem poderes e condutas especiais sobre o meio ambiente, haja vista que leis sustentam seus direitos, assim como a natureza é entendida como fundamental para sustentação de sua cultura, aspectos estes que podem ser vinculados à gestão em prol da conservação dos recursos naturais. Para isso, é necessário conhecer legislações e saber conciliar conflitos por assimetrias, como forte ativismo e interesses de grupos considerados hegemônicos (Richmond et al., 2013). 
Diante disto, percebe-se que a eficiência de uma gestão ambiental está diretamente relacionada com os meios e processos utilizados para compreender as condutas humanas e flexioná-las em benefício do meio ambiente natural, como também para provento da humanidade.

\section{Fatores que afetam o comportamento ambiental de residentes}

A base de uma sociedade equilibrada está diretamente relacionada com a forma como as pessoas se conduzem mediante as situações que estão ao seu entorno, no modo como se relacionam com os familiares e vizinhos e tratam de problemas políticos e ideológicos, bem como se comportam perante o meio ambiente (artificial, natural e cultural).

Quando se trata de planejamento de regiões turísticas, esse equilíbrio volta-se significativamente para participação e/ou responsabilidade social. A participação da comunidade, no âmbito do planejamento turístico, pode implicar diversos benefícios, dentre eles, melhor confiança nas decisões, aceitação pública das decisões propostas e compreensão dos projetos, integração dos vários interesses e opiniões, assim como promoção e desenvolvimento da aprendizagem social (Luyet, Schlaepfer, Parlange, \& Buttler, 2012).

No caso de destinos turísticos costeiros, o planejamento da atividade deve ainda ser mais detalhado, devido a sua relevância ecológica, já que são áreas ambientalmente sensíveis, caracterizadas por ecossistemas diferenciados e biodiversidade, constituindo-se em um dos espaços mais impactados pela ação antrópica. Para isso, entende-se como necessário que os próprios residentes adotem um comportamento ambiental, ou seja, conduzido por boas práticas de preservação e conservação de recursos naturais.

Segundo Corral-Verdugo e Pinheiro (1999), as primeiras investigações sobre comportamento ambiental foram orientadas por dois campos de estudos: o behaviorista, indicando que o comportamento pró-ambiental é afetado por fatores como castigos, reforçadores (positivos e negativos), antecedentes ou consequentes da conduta ambiental, considerados fatores externos a ele; e o cognitivista, inferindo que o esse comportamento sofre influência de variáveis internas dos indivíduos, como atitudes, conhecimentos e personalidade.

$\mathrm{O}$ uso de tais fatores pode ser observado em diversos estudos. Deboni, Mombach, Lopes e Simioni (2015) avaliaram a percepção ambiental de residentes de Lages, SC, Brasil, quanto aos aspectos ambientais vivenciados no local. Os autores utilizaram como variáveis o destino de resíduos sólidos (uso de sacolas plásticas, descartes eletrônicos e lâmpadas inutilizadas e queima de lixo doméstico), bem como destinação de óleo de cozinha, consumo de energia e uso e destino de água e efluentes. Pôde-se verificar a relação do nível de escolaridade e gênero com a preocupação e conscientização ambiental, pois pessoas com idade maior e mulheres demostraram percepção mais consciente no que concerne às questões ambientais.

Lamano-Ferreira, Morizono, Ribeiro, Kniess e Ramos (2015), ao tentarem representar o comportamento ecológico/ambiental de moradores de São Paulo, SP, Brasil, por meio de atitudes ambientais, utilizaram a economia de recursos naturais, limpeza urbana, reciclagem e ativismo-consumo como variáveis de avaliação. Revelou-se que atitudes ligadas ao lixo urbano e reciclagem foram as mais 
ressaltadas entre os entrevistados. Ademais, verificou-se na dimensão ativismo-consumo tendências para o uso de produtos ecológicos e embalagens reutilizáveis, assim como para o uso de bicicleta como meio de transporte.

Observam-se também estudos que utilizaram o aspecto de arborização como meio de investigação. Araújo, Araújo e Araújo (2010) verificaram a percepção ambiental de moradores do bairro Presidente Médici, em Campina Grande, PB, Brasil, em relação à arborização local, a partir do uso das seguintes variáveis: número de moradores por residência, escolaridade, nível de arborização da rua, importância da arborização, fatores positivos e negativos da arborização, colaboração para com a arborização e conhecimentos de espécies para plantio. Os autores avaliaram que, independentemente do nível de escolaridade, os entrevistados tinham conhecimento dos benefícios gerados pelo processo em questão e da importância deste para qualidade de vida nas cidades. Os aspectos negativos identificados voltaram-se para a escolha e manejo das espécies que seriam plantadas, problemas estes que, segundo os autores, poderiam ser repelidos com ações educativas.

Percebe-se que estudos do comportamento ambiental podem englobar inúmeras dimensões e variáveis, bem como os resultados podem distinguir-se de acordo com amostragem e contexto. Sendo assim, sua investigação permite a utilização de diferentes meios e processos. Por se tratar de conduta humana, pressupõe-se que o comportamento ambiental possa ser inferido a partir do comportamento de compra, passível de ser estudado pela teoria do comportamento planejado (TCP), uma das mais utilizadas para analisar a relação intenção-comportamento (Davies, Foxall, \& Pollister, 2002).

Criada por Ajzen em 1985, a TCP originou-se da teoria da ação racional (TAR) com o propósito de prever comportamentos em contextos diferentes a partir de um número reduzido de variáveis explicáveis (Ajzen, 1991; Huang, 2011). A TCP propõe que a intenção comportamental, principal antecedente do comportamento sofre influências da atitude (avaliação favorável ou desfavorável em relação ao comportamento), normas subjetivas (percepção resultante da pressão social exercida sobre essa conduta) e controle comportamental percebido (percepção do controlo sobre a ação) (Santos, Veiga, \& Moura, 2010). Apesar de ser uma teoria com respaldo, verificam-se, em literatura, estudos que adicionam novos construtos ao modelo original, como comportamento passado (Moura, Veiga, Cunha, \& Moura, 2012; Sharifirad, Yarmohammadi, Azadbakht, Morowatisharifabad, \& Hassanzadeh, 2013) e segurança (Santos et al., 2010), com o intuito de atestar sua efetividade em diversos contextos, como o ambiente profissional (Van Hooft \& De Jong, 2009, Wanberg, Glomb, Song, \& Sorenson, 2005), escolha de carreira (Caska, 1998; Tang, Fouad, \& Smith, 1999), consumo de alimentos (Dunn, Mohr, Wilson, \& Wittert, 2011) e compras via internet (Straub \& Watson, 2001).

As variáveis direcionadoras de comportamento propostas pela TCP têm caráter eminentemente cognitivo. Para fins de comportamento ambiental, pressupõe-se a necessidade da investigação de variáveis de caráter afetivo, como direcionadoras de comportamento, tal como proposto nos estudos de Bonaiuto, Carrus, Martorella e Bonnes (2002), Walker e Ryan (2008) e Buijs (2009). Em termos de caráter afetivo, uma das principais variáveis estudadas é "apego ao lugar" (place attachment), identificado com um vínculo afetivo e emocional entre o indivíduo e sua comunidade (Lee, 2013), emergindo como um conceito central em diversos estudos geográficos e ambientais (Walker \& Ryan, 2008). 
"Apego ao lugar" engloba afetos e emoções entre pessoas e os lugares, porém este lugar deve garantir a satisfação de necessidades, o valor simbólico do lugar para as pessoas, a permanência desta relação, ou mesmo a mobilidade quando é necessário, entre outros. Sendo assim, diversos fatores podem interferir nesse tipo de afeto, como violência urbana, poluição (sonora, visual, de águas etc.), desemprego, condições de moradia entre outros (Lima \& Bomfim, 2009). Esse sentimento ocorre nos indivíduos cuja identidade de lugar envolve cognições positivamente valorizadas de uma ou alguma combinação desses contextos, que de longe pesam mais que os números de cognições negativamente valorizadas (Proshansky, Fabian, \& Kaminoff, 1983, citado por Felippe \& Kuhnen, 2012).

0 vínculo afetivo e emocional com o lugar é verificado como um fator influente capaz de efetivar de modo mais equitativo diversas atividades, mormente no apoio dos residentes ao desenvolvimento do turismo (Lee, 2013). Diante disso, observa-se que um indivíduo que possui forte apego ao lugar, além de prezar por questões sociais e econômicas mais equitativas, está mais propenso a aderir a uma postura ecologicamente mais responsável. 0 apego ao lugar tem sido considerado uma variável antecedente à satisfação, segundo Hwang, Lee e Chen (2005), ao comportamento pró-ambiental, segundo Walker e Ryan (2008) ou lealdade (fidelidade), segundo Yuksel, Yuksel e Bilim (2010).

Baseando-se nos conceitos apresentados, propôs-se o estudo cujas características metodológicas são apresentadas a seguir:

\section{METODOLOGIA}

O presente estudo utilizou-se do método hipotético-dedutivo, uma vez que se apropriou de alguns modelos teóricos para chegar à construção de hipóteses e assim elencar os possíveis fatores que poderiam afetar o comportamento ambiental de residentes em destinos turísticos costeiros. Com relação à abordagem, caracteriza-se como quantitativo, uma vez que o problema de pesquisa foi investigado por meio de métodos quantificáveis, tanto nas modalidades de coleta de dados quanto no tratamento destes por meio de técnicas estatísticas. Em relação aos objetivos, é descritivo.

0 estudo foi realizado no município de Baía Formosa (coordenadas geográficas $06^{\circ} 22^{\prime} 08^{\prime \prime} \mathrm{S}$ e $35^{\circ} 00^{\prime} 28^{\prime \prime} \mathrm{W}$ ), destino turístico costeiro em consolidação, localizado no litoral sul do estado do Rio Grande do Norte, Brasil, a 98,9 Km de Natal, capital do estado. A localidade abrange uma área de $245,661 \mathrm{Km}^{2}$, na qual se pode encontrar uma grande diversidade de atrativos naturais, como dunas, falésias, manguezal e lagoas. A população do município é estimada em 9.247 habitantes (Instituto Brasileiro de Geografia e Estatística [IBGE], 2017), sendo entrevistados 288 residentes. 0 tipo de amostragem utilizada foi a não probabilística, tendo em vista que não se mensurou a probabilidade de os investigados serem incluídos na pesquisa.

0 instrumento de pesquisa utilizado foi o questionário, constituído por variáveis baseadas na TCP, teoria que expõe que o comportamento humano é precedido pela intenção comportamental. Esta, neste estudo, foi concebida como intenção ambiental, influenciada pelos seguintes constructos: atitude pró-ambiental (grau de favorabilidade e desfavorabilidade dos sujeitos em relação ao meio ambiente), normas subjetivas (pressão social sobre o comportamento ambiental 
das pessoas) e controle comportamental percebido (força de fatores além do controle volitivo dos investigados) (Ajzen, 1991).

Além disso, foi adicionado ao modelo, o constructo "apego ao lugar" (afeto dos investigados pelo seu local de residência), visto que esse constructo apresenta influência sobre o comportamental ambiental (Bonaiuto et al., 2002; Walter \& Ryan, 2008; Buijs, 2009; Lee, 2013). As variáveis componentes dessas dimensões foram avaliadas por meio de uma escala métrica de onze pontos (baseada na estrutura Likert), a qual variou entre 0 (discordo plenamente) a 10 (concordo plenamente), conforme uma frase proposta que representava a variável (Quadro 1).

Quadro 1 - Variáveis e dimensões utilizadas no estudo

\begin{tabular}{|c|c|c|}
\hline Dimensão & Variável & Descrição da variável \\
\hline \multirow{3}{*}{$\begin{array}{l}\text { Atitude pró- } \\
\text {-ambiental }\end{array}$} & AT1 & $\begin{array}{l}\text { Eu acho muito importante realizar ações para proteger o } \\
\text { meio ambiente. }\end{array}$ \\
\hline & AT2 & $\begin{array}{l}\text { Proteger o meio ambiente gera uma sensação de bem- } \\
\text { estar na pessoa. }\end{array}$ \\
\hline & AT3 & $\begin{array}{l}\text { Eu acho uma boa ideia realizar ações para proteger o } \\
\text { meio ambiente. }\end{array}$ \\
\hline \multirow{3}{*}{ Normas subjetivas } & NSUB1 & $\begin{array}{l}\text { As pessoas que são importantes para mim acham que } \\
\text { seria importante eu proteger o meio ambiente. }\end{array}$ \\
\hline & NSUB2 & $\begin{array}{l}\text { As pessoas que influenciam minhas decisões acham que } \\
\text { seria importante eu proteger o meio ambiente. }\end{array}$ \\
\hline & NSUB3 & $\begin{array}{l}\text { No meu círculo de amizade, as pessoas acham que seria } \\
\text { importante eu proteger o meio ambiente. }\end{array}$ \\
\hline \multirow{3}{*}{$\begin{array}{l}\text { Controle } \\
\text { percebido }\end{array}$} & CONT1 & $\begin{array}{l}\text { Eu tenho conhecimento para ajudar a proteger o meio } \\
\text { ambiente. }\end{array}$ \\
\hline & CONT2 & $\begin{array}{l}\text { Só depende de mim realizar ações para proteger o meio } \\
\text { ambiente. }\end{array}$ \\
\hline & CONT3 & $\begin{array}{l}\text { Não preciso de qualquer ação da prefeitura para ter } \\
\text { comportamento ambiental. }\end{array}$ \\
\hline \multirow{3}{*}{$\begin{array}{l}\text { Intenção pró- } \\
\text {-ambiental }\end{array}$} & INT1 & Eu tenho vontade de ajudar a proteger o meio ambiente. \\
\hline & INT2 & $\begin{array}{l}\text { Eu pretendo aumentar minhas ações para proteger o meio } \\
\text { ambiente. }\end{array}$ \\
\hline & INT3 & $\begin{array}{l}\text { Se for possível, vou melhorar minhas ações para proteger } \\
\text { o meio ambiente. }\end{array}$ \\
\hline \multirow{4}{*}{ Apego ao lugar } & APEG1 & Possuo um grande apego à Baía Formosa. \\
\hline & APEG2 & Sinto Baía Formosa como uma parte de mim. \\
\hline & APEG3 & Me identifico fortemente com Baía Formosa. \\
\hline & APEG4 & Estou muito satisfeito em morar em Baía Formosa. \\
\hline \multirow{3}{*}{$\begin{array}{l}\text { Comportamento } \\
\text { pró-ambiental }\end{array}$} & CAM1 & $\begin{array}{l}\text { Considero que realizo ações para proteger o meio } \\
\text { ambiente. }\end{array}$ \\
\hline & CAM2 & $\begin{array}{l}\text { Sempre quando há condições, procuro ajudar a proteger o } \\
\text { meio ambiente. }\end{array}$ \\
\hline & CAM3 & Procuro economizar água em minha casa. \\
\hline
\end{tabular}

Fonte - Dados da pesquisa, 2017 
Após a elaboração do questionário, foi realizado um pré-teste com os residentes a fim de avaliar a qualidade geral do instrumento no que se refere à clareza do conteúdo, organização e tempo médio de respostas, bem como identificar possíveis entraves que pudessem interferir no andamento da pesquisa. A aplicação final do questionário ocorreu entre março e abril do ano corrente, 2017.

0 modelo de análise é apresentado na Figura 1, sendo constituído pelas seguintes dimensões: atitude pró-ambiental, normas subjetivas, controle percebido, intenção pró-ambiental, apego ao lugar e comportamento pró-ambiental.

Figura 1 - Modelo estrutural para investigar o comportamento ambiental de residentes

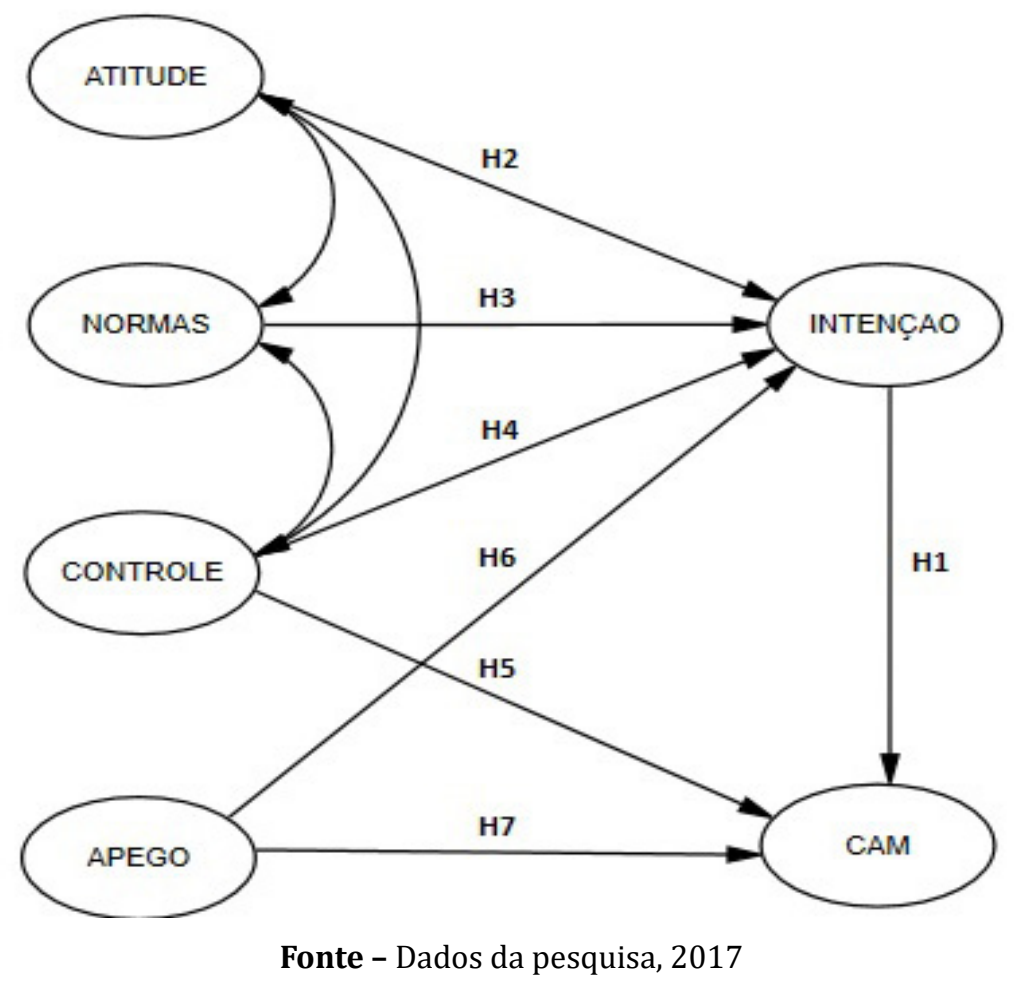

As hipóteses testadas a partir deste modelo são:

H1: Existe significativa correlação entre "intenção ambiental" e "comportamento ambiental"

H2: Existe significativa correlação entre "atitude pró-ambiental" e "intenção ambiental"

H3: Existe significativa correlação entre "normas subjetivas" e "intenção ambiental"

H4: Existe significativa correlação entre "controle comportamental percebido" e "intenção ambiental".

H5: Existe significativa correlação entre "controle comportamental percebido" e "comportamento ambiental"

H6: Existe significativa correlação entre "apego ao lugar" e "intenção pró-ambiental"

H7: Existe significativa correlação entre "apego ao lugar" e "comportamento ambiental". 
Os dados coletados foram analisados pelo método de modelagem de equações estruturais, aportado pelo Statistical Package for the Social Sciences (SPSS), versão 22 para Windows, com pacote Analysis of Moment Structures (AMOS). Para realizar esse procedimento, inicialmente foi necessária a composição das dimensões, realizada por meio do método de análise fatorial. A modelagem de equações estruturais refere-se a uma técnica de modelação generalizada, utilizada para testar a validade de modelos teóricos que definem relações casuais hipotéticas entre variáveis. Essas relações são representadas por parâmetros que indicam a magnitude do efeito que as variáveis, ditas independentes, apresentam sobre outras variáveis, ditas dependentes, num conjunto de hipóteses respeitantes a padrões de associação entre as variáveis no modelo (Marôco, 2010, p. 3).

\section{RESULTADOS}

\section{Adequação de um modelo de mensuração}

O modelo de mensuração (também conhecido como submodelo de medida) define a forma como os constructos ou dimensões hipotéticas são operacionalizados pelas variáveis observadas ou manifestas (Marôco, 2010, p. 17). Para compor um modelo de mensuração adequado para investigar as inter-relações existentes entre as variáveis propostas neste estudo, inicialmente foi utilizada a análise fatorial exploratória (AFE). Esta técnica estatística permite que, a partir de um conjunto de variáveis observáveis (questões do instrumento de pesquisa), sejam compostas dimensões de variabilidades comuns, em um conjunto de fenômenos (Corrar, Paulo, \& Dias, 2009). Para constituição dessas dimensões, foram utilizados os seguintes indicadores da análise fatorial: comunalidade e carga fatorial de cada variável da respectiva dimensão, o teste Kaiser-Meyer-Olkin (KMO) e a variância total explicada de cada constructo, assim como o coeficiente alfa de Cronbach, referente a uma análise de confiabilidade do questionário, para cada dimensão proposta.

Comunalidade representa a quantia total de variância que uma variável original compartilha com todas as outras incluídas na análise. Assume-se que os valores de referência desse comunalidade para que cada variável contribua na constituição da dimensão deva ser igual ou maior que 0,6 (Hair Junior, Anderson, Tatham, \& Black, 1999). Carga fatorial são valores que medem o grau de correlação entre as variáveis originais e os fatores (Corrar et al., 2009). A carga fatorial deve exceder 0,70 para que o fator possa explicar $50 \%$ da variância e tenha significância prática (Hair Junior et al. 1999). 0 teste KMO mede o grau de correlação parcial entre as variáveis. Valores próximos a 1,0 para o KMO são considerados adequados para estudos desta natureza (Corrar et al., 2009).

No caso da variância total explicada, estabelece-se o comportamento dos fatores e sua efetiva combinação como indicador da variância observada, ou seja, explica-se a porcentagem da variação da variável dependente (fator), esclarecida pela variância das variáveis independentes (observáveis). 0 indicador de confiabilidade alfa de Cronbach mede a correlação entre respostas em um questionário através da análise do perfil das respostas dadas pelos respondentes. Dado que 
todos os itens de um questionário utilizam a mesma escala de medição, o coeficiente $\alpha$ é calculado a partir da variância dos itens individuais e da variância da soma dos itens de cada avaliador. Ele varia de 0 a 1, sendo os valores de 0,6 a 0,7 considerados o valor mínimo de aceitabilidade. Porém, o valor 0,6 é aceito para pesquisas exploratórias (Hair Junior et al. 1999). A Tabela 1 apresenta os indicadores da AFE para as variáveis utilizadas no estudo:

Tabela 1 - Indicadores da análise fatorial exploratória

\begin{tabular}{|c|c|c|c|c|c|c|}
\hline Dimensão & Variável & $\begin{array}{l}\text { Carga } \\
\text { fatorial }\end{array}$ & Comunalidade & KMO & $\begin{array}{l}\text { Variância } \\
\text { total } \\
\text { explicada }\end{array}$ & $\begin{array}{c}\text { Alfa de } \\
\text { Cronbach }\end{array}$ \\
\hline \multirow{3}{*}{$\begin{array}{l}\text { Atitude pró- } \\
\text {-ambiental }\end{array}$} & AT1 & 0,901 & 0,811 & \multirow{3}{*}{0,739} & \multirow{3}{*}{82,779} & \multirow{3}{*}{0,896} \\
\hline & AT2 & 0,899 & 0,808 & & & \\
\hline & AT3 & 0,930 & 0,864 & & & \\
\hline \multirow{3}{*}{$\begin{array}{l}\text { Normas } \\
\text { subjetivas }\end{array}$} & NSUB1 & 0,931 & 0,866 & \multirow{3}{*}{0,765} & \multirow{3}{*}{87,941} & \multirow{3}{*}{0,921} \\
\hline & NSUB2 & 0,945 & 0,892 & & & \\
\hline & NSUB3 & 0,938 & 0,880 & & & \\
\hline \multirow{3}{*}{$\begin{array}{l}\text { Controle } \\
\text { percebido }\end{array}$} & CONT1 & 0,743 & 0,552 & \multirow{3}{*}{0,661} & \multirow{3}{*}{67,299} & \multirow{3}{*}{0,752} \\
\hline & CONT2 & 0,860 & 0,740 & & & \\
\hline & CONT3 & 0,852 & 0,726 & & & \\
\hline \multirow{3}{*}{$\begin{array}{l}\text { Intenção pró- } \\
\text {-ambiental }\end{array}$} & INT1 & 0,795 & 0,632 & \multirow{3}{*}{0,689} & \multirow{3}{*}{70,874} & \multirow{3}{*}{0,792} \\
\hline & INT2 & 0,873 & 0,763 & & & \\
\hline & INT3 & 0,855 & 0,732 & & & \\
\hline \multirow{4}{*}{ Apego ao lugar } & APEG1 & 0,910 & 0,828 & \multirow{4}{*}{0,843} & \multirow{4}{*}{82,899} & \multirow{4}{*}{0,931} \\
\hline & APEG2 & 0,925 & 0,855 & & & \\
\hline & APEG3 & 0,913 & 0,833 & & & \\
\hline & APEG4 & 0,895 & 0,801 & & & \\
\hline \multirow{3}{*}{$\begin{array}{c}\text { Comportamento } \\
\text { pró-ambiental }\end{array}$} & CAM1 & 0,829 & 0,688 & \multirow{3}{*}{0,638} & \multirow{3}{*}{68,465} & \multirow{3}{*}{0,764} \\
\hline & CAM2 & 0,892 & 0,796 & & & \\
\hline & CAM3 & 0,755 & 0,570 & & & \\
\hline
\end{tabular}

Fonte - Dados da Pesquisa, 2017

Conforme pode ser observado na Tabela 1, o conjunto de variáveis selecionadas pode ser considerado como adequado para compor cada dimensão respectiva, visto que, em todas as dimensões, os indicadores da análise fatorial apresentaram valores dentro do aceitável, tendo como base os parâmetros-limites considerados. Observa-se que na dimensão "controle percebido", os indicadores da análise fatorial apresentaram valores muito próximos ao limite, influenciados pela carga fatorial da variável CONT1 (Eu tenho conhecimento para ajudar a proteger o meio ambiente). Tal fato, contribui para a obtenção de uma menor combinação de variáveis explicativas da variância observada $(67,299)$. Entretanto, o indicador alfa de Cronbach dessa dimensão $(0,752)$ indica uma 
razoável correlação entre as respostas do questionário, possibilitando então o uso da variável CONT1 como componente da dimensão "controle percebido". Tal fato também ocorre com a variável CAM3 (Procuro economizar água em minha casa) da dimensão "comportamento pró-ambiental", mas ainda dentro dos limites considerados aceitáveis para a composição da dimensão.

\section{Validação dos constructos do modelo}

0 objetivo deste procedimento foi apresentar a validação dos constructos do modelo. 0 método de validação utilizou os indicadores de confiabilidade composta do constructo e variância extraída (AVE). A confiabilidade composta sinaliza a consistência interna dos indicadores que compõem o construto (Hair Junior et al., 1999). Já a AVE é uma medida que, com base na confiabilidade dos construtos, sinaliza a confiabilidade do modelo (Hair Junior et al., 2009). 0 valor mínimo aceitável para a confiabilidade composta é 0,70 e o considerado básico para a variância extraída é 0,50 (Hair Junior et al., 1999). 0 cálculo desses indicadores possui a seguinte formulação:

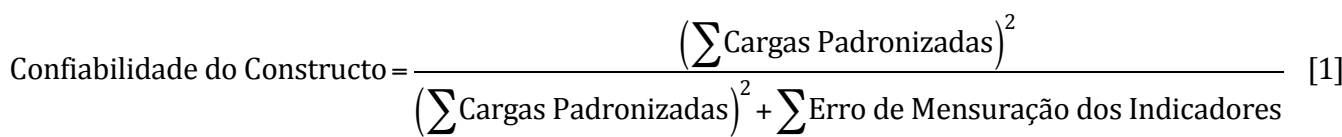

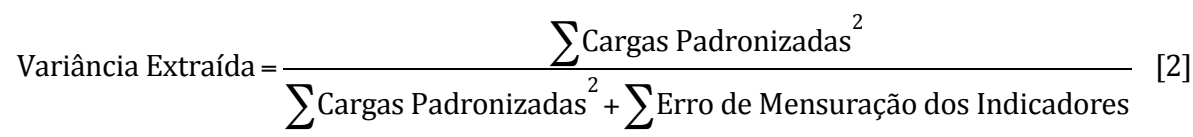

O erro de mensuração dos indicadores é calculado a partir da seguinte relação matemática:

Erro $=1-(\text { Carga Padronizada })^{2}[3]$

Na Tabela 2 são apresentados os resultados da confiabilidade composta do constructo e da AVE do modelo.

Conforme pode ser observado pelos dados apresentados na Tabela 2, todos os constructos utilizados no modelo de mensuração apresentam consistência interna aceitável, já que o valor da confiabilidade composta foi superior ao de referência $(0,70)$. Análise semelhante pode ser remetida à avaliação da variância extraída do modelo de mensuração, cujos indicadores em todos os constructos foram superiores ao critério adotado $(0,5)$, demonstrando a confiabilidade do modelo proposto. 
Tabela 2 - Estimativa dos valores de confiabilidade composta e variância extraída dos constructos

\begin{tabular}{|c|c|c|c|c|c|c|c|}
\hline Constructo & $\begin{array}{l}\text { Variáveis } \\
\text { (relações } \\
\text { lineares) }\end{array}$ & $\begin{array}{c}\text { Carga } \\
\text { não } \\
\text { padronizada }\end{array}$ & $\begin{array}{c}\text { Erro } \\
\text { padrão }\end{array}$ & C. R. & $\begin{array}{c}\text { Carga } \\
\text { padronizada }\end{array}$ & $\mathbf{p}$ & $\begin{array}{l}\text { Confiabilidade } \\
\text { composta } \\
\text { variância } \\
\text { extraída }\end{array}$ \\
\hline \multirow{3}{*}{ Atitude } & AT1<---ATITUDE & 1,000 & - & - & 0,840 & $* * *$ & \multirow{3}{*}{$\begin{array}{c}\text { Confiabilidade: } \\
0,897 \\
\text { AVE: } 0,745\end{array}$} \\
\hline & AT2<---ATITUDE & 0,992 & 0,060 & 16,558 & 0,832 & $* * *$ & \\
\hline & AT3<---ATITUDE & 1,164 & 0,063 & 18,847 & 0,915 & $* * *$ & \\
\hline \multirow{3}{*}{ Normas } & NSUB1<---NORMAS & 1,000 & - & - & 0,883 & $* * *$ & \multirow{3}{*}{$\begin{array}{c}\text { Confiabilidade: } \\
0,931 \\
\text { AVE: } 0,819\end{array}$} \\
\hline & NSUB2<---NORMAS & 1,054 & 0,046 & 22,913 & 0,919 & $* * *$ & \\
\hline & NSUB4<---NORMAS & 1,063 & 0,048 & 22,314 & 0,913 & $* * *$ & \\
\hline \multirow{3}{*}{ Controle } & $\begin{array}{l}\text { CONT2<--- } \\
\text { CONTROLE }\end{array}$ & 1,000 & - & - & 0,613 & $* * *$ & \multirow{3}{*}{$\begin{array}{c}\text { Confiabilidade: } \\
0,764 \\
\text { AVE: } 0,522\end{array}$} \\
\hline & $\begin{array}{l}\text { CONT5<--- } \\
\text { CONTROLE }\end{array}$ & 1,841 & 0,205 & 8,958 & 0,800 & $* * *$ & \\
\hline & $\begin{array}{l}\text { CONT6<--- } \\
\text { CONTROLE }\end{array}$ & 1,779 & 0,201 & 8,873 & 0,743 & $* * *$ & \\
\hline \multirow{4}{*}{ Apego } & APEG3<---APEGO & 1,000 & - & - & 0,843 & $* * *$ & \multirow{4}{*}{$\begin{array}{c}\text { Confiabilidade: } \\
0,926 \\
\text { AVE: } 0,758\end{array}$} \\
\hline & APEG4<---APEGO & 1,078 & 0,048 & 22,697 & 0,870 & $* * *$ & \\
\hline & APEG5<---APEGO & 1,190 & 0,203 & 5,854 & 0,900 & $* * *$ & \\
\hline & APEG6<---APEGO & 1,071 & 0,184 & 5,813 & 0,868 & $* * *$ & \\
\hline \multirow{3}{*}{ Intenção } & INT1<---INTENÇAO & 1,000 & - & - & 0,782 & $* * *$ & \multirow{3}{*}{$\begin{array}{c}\text { Confiabilidade: } \\
0,832 \\
\text { AVE: } 0,625\end{array}$} \\
\hline & INT2<---INTENÇÃO & 1,491 & 0,124 & 12,013 & 0,889 & $* * *$ & \\
\hline & INT3<---INTENÇÃO & 1,095 & 0,100 & 10,416 & 0,688 & $* * *$ & \\
\hline \multirow{3}{*}{ CAM } & CAM1<---CAM & 1,000 & - & - & 0,796 & $* * *$ & \multirow{3}{*}{$\begin{array}{c}\text { Confiabilidade: } \\
0,749 \\
\text { AVE: } 0,510\end{array}$} \\
\hline & CAM2<---CAM & 0,881 & 0,066 & 13,268 & 0,814 & $* * *$ & \\
\hline & САM3<---CAM & 0,490 & 0,066 & 7,427 & 0,483 & $* * *$ & \\
\hline
\end{tabular}

Fonte - Pesquisa de campo (2017)

Notas: CAM - Comportamento pró-ambiental ${ }^{* * *}$ Relação altamente significativa

\section{Construção do modelo estrutural}

0 modelo estrutural define as relações causais ou de associação entre as dimensões propostas (Marôco, 2010:19), objeto principal deste estudo. Para que sejam plausíveis as relações propostas, há a necessidade do atendimento de alguns pressupostos da técnica estatística. Um dos pressupostos é a necessidade da ausência de multicolinearidade, ou seja, na parte estrutural do modelo, as variáveis exógenas ou independentes não podem estar fortemente associadas (Marôco, 2010:62). Para verificar tal pressuposto, realizou-se a análise de correlação bivariada $\left(\mathrm{r}_{\mathrm{c}}\right)$ entre as dimensões obtidas a partir da definição do modelo de mensuração do estudo. Os resultados são apresentados a seguir: 
Análise de correlações bivariadas $\left(r_{c}\right)$

Na Figura 2 é apresentada a análise de correlação bivariada entre as variáveis exógenas (independentes) e endógenas (dependentes) do modelo proposto:

Figura 2 - Análise da Correlação Bivariada

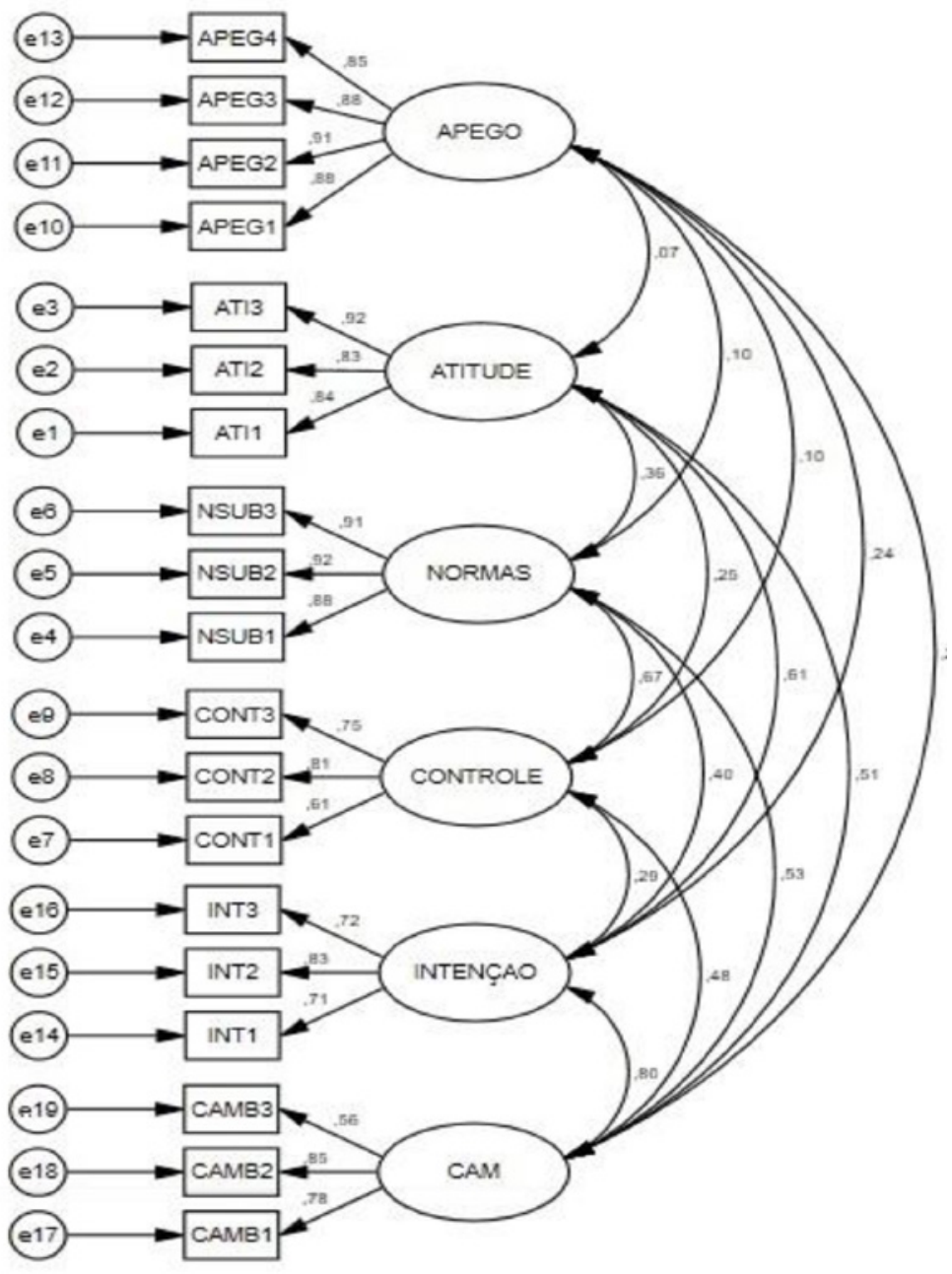

Fonte - Dados do Campo (2017)

Conforme pode ser observado através dos coeficientes de correlação bivariada, apresentados na Figura 2, não se identificam correlações bivariadas fortes entre as variáveis exógenas do modelo. Observam-se correlações moderadas entre as dimensões "atitude e normas" $\left(\mathrm{r}_{\mathrm{c}}\right.$ de 0,36$)$ e "normas e controle percebido" $\left(r_{c}\right.$ de 0,67$)$, neste caso, tendendo a uma correlação bivariada forte. Ambas as correlações já foram identificadas por Ajzen (1991) e fazem parte do modelo estrutural básico dos fatores que visam explicar o comportamento. Com relação à variável exógena "apego", observam-se correlações bivariadas fracas com as demais variáveis exógenas, o que indica baixa associação entre as dimensões e a existência de um baixo nível de multicolinearidade.

Verificam-se relações de correlação altas entre variáveis exógenas e endógenas (o que não indica multicolinearidade), principalmente entre "intenção pró-ambiental" e "comportamento pró-ambiental" $\left(r_{c}\right.$ de 0,80$)$ e relação fraca entre "apego" e "comportamento pró-ambiental" ( $r_{c}$ de 0,27$)$, no que se pode inferir 
que o componente afetivo do modelo influi de maneira fraca no comportamento pró-ambiental.

Assumindo-se então a existência de multicolinearidade forte entre as variáveis exógenas do modelo, propôs-se analisar o modelo estrutural obtido, cujas considerações são apresentadas a seguir.

\section{Análise do modelo estrutural}

Na Figura 3 é apresentado o modelo estrutural obtido a partir das hipóteses de relacionamento propostas:

Figura 3 - Modelo estrutural do estudo

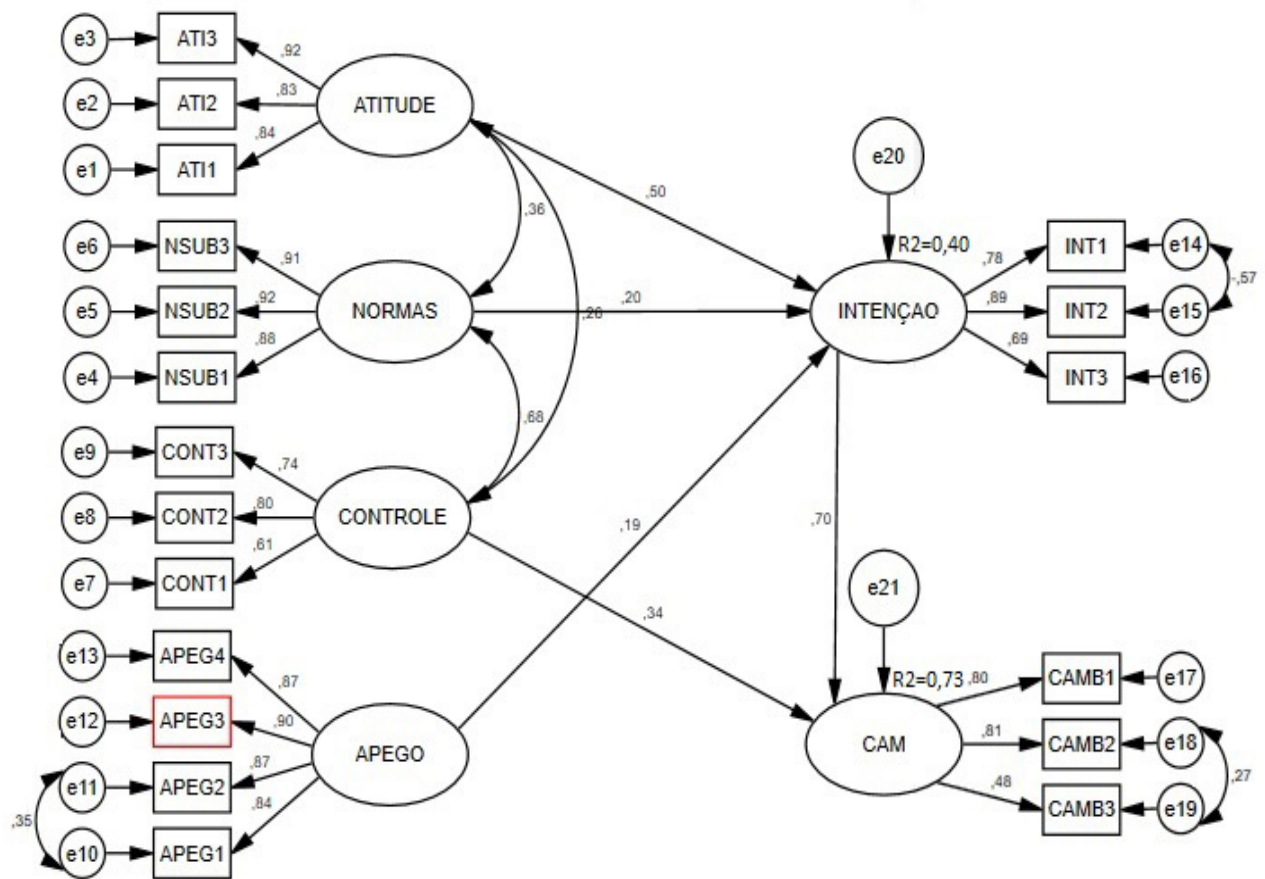

Fonte - Pesquisa de campo (2017)

As relações causais entre "controle" e "intenção pró-ambiental" ( $p=0,841)$ e "apego" e "comportamento pró-ambiental" $(p=0,089)$ foram consideradas como não significativas (p significa a probabilidade de erro de se assumir relação causal significativa entre as variáveis). No caso deste estudo, assume-se relações causais significativas quando $\mathrm{p}<0,05$ ). Demais trilhas foram consideradas significativas.

A partir dos coeficientes de trilha apresentados na Figura 3, observa-se uma relação estrutural adequada principalmente quando se verifica o valor do $\mathrm{R}^{2}$ obtido $(0,73)$ com a variável dependente "comportamento pró-ambiental" (CAM) e o $\mathrm{R}^{2}$ obtido $(0,40)$ com a variável dependente "intenção pró-ambiental" (intenção). Nota-se , assim, uma relação causal entre as dimensões dependentes "intenção" e "comportamento pró-ambiental". Observa-se relação fraca (coeficiente de trilha de $0,19)$ entre a dimensão "apego ao lugar" (apego) e "intenção" e relação não significativa entre "apego" e a variável dependente CAM.

De maneira geral, verifica-se que o componente cognitivo do modelo, composto pelas variáveis definidas a partir da TCP apresentam maior poder 
explicativo, tanto de intenção ambiental quanto comportamento pró-ambiental, do que o componente afetivo do modelo, representado pela variável independente "apego ao lugar".

Para que seja verificado quão bem o modelo teórico proposto é capaz de reproduzir uma estrutura correlacional das variáveis observáveis na amostra sob estudo, há a necessidade da avaliação da qualidade de ajustamento do modelo. Neste estudo, essa avaliação é realizada a partir de índices empíricos que se baseiam nas funções de verossimilhança ou matriz dos resíduos obtidos durante o ajustamento do modelo (Marôco, 2010, p. 40). Na Tabela 3 são apresentados os índices de qualidade de ajustamento do modelo:

Tabela 3 - Índices de qualidade de ajustamento do modelo

\begin{tabular}{|c|c|c|c|}
\hline Índices & Grupo do índice & Resultados & $\begin{array}{l}\text { Valores de referência } \\
\text { (Marôco, 2010) }\end{array}$ \\
\hline $\begin{array}{l}\text { TLI } \\
\text { CFI } \\
\text { NFI }\end{array}$ & Índices relativos & $\begin{array}{l}0,958 \\
0,966 \\
0,929\end{array}$ & $\begin{array}{l}{[0,90-0,95 \text { [Ajustamento bom }} \\
>0,95 \text { Ajustamento muito bom }\end{array}$ \\
\hline $\begin{array}{l}\text { PCFI } \\
\text { PGFI }\end{array}$ & Índice de parcimônia & $\begin{array}{l}0,778 \\
0,672\end{array}$ & [0,6 - 0,8 [Ajustamento razoável \\
\hline RMSEA & $\begin{array}{l}\text { Índice de discrepância } \\
\text { populacional }\end{array}$ & 0,050 & $<0.05$ - Ajustamento bom \\
\hline $\begin{array}{l}\mathrm{X} 2 / \mathrm{DF} \\
\mathrm{GFI}\end{array}$ & Índices absolutos & $\begin{array}{l}1,853 \\
0,914\end{array}$ & $\begin{array}{c}\text { ]1 - 2] Ajustamento bom } \\
{[0,90-0,95 \text { [Ajustamento bom }}\end{array}$ \\
\hline
\end{tabular}

Fonte - Pesquisa de Campo (2017)

Observa-se que o modelo teórico proposto, estimado a partir dos dados coletados, apresentam índices de qualidade de ajustamento entre bons e muito bons, de acordo com os valores de referência propostos, a não ser nos índices de Parcimônia (PCFI e PGFI) que apresentaram resultados razoáveis. Destaca-se que o objetivo dos índices de parcimônia é compensar a melhoria artificial do modelo que se consegue, simplesmente pela inclusão de mais parâmetros livres aproximando o modelo sob estudo de um modelo saturado (Marôco, 2010:46), o que pode ter acontecido quando da introdução da dimensão Apego ao Lugar ao modelo original proposto.

\section{Análise das Hipóteses}

Com base nos resultados encontrados, para o grupo de entrevistados utilizados como objeto da pesquisa, pode-se concluir em relação às hipóteses propostas:

H1: Existe significativa correlação positiva entre "intenção ambiental" e "comportamento ambiental".

H2: Existe significativa correlação positiva entre "atitude pró-ambiental" e "intenção ambiental".

H3: Existe significativa correlação positiva entre "normas subjetivas" e "intenção ambiental".

H4: Não existe correlação significativa entre "controle comportamental percebido" e "intenção ambiental". 
H5: existe significativa correlação positiva entre "controle comportamental percebido" e "comportamento ambiental".

H6: Não existe correlação significativa entre "apego ao lugar" e "comportamento ambiental".

H7: Existe significativa correlação positiva entre "apego ao lugar" e "intenção pró-ambiental".

\section{CONCLUSÕES}

O objetivo do estudo foi analisar as inter-relações entre fatores capazes de influenciar o comportamento ambiental de residentes. Para tanto, foi necessário inicialmente adequar um modelo de mensuração capaz de investigar o relacionamento das variáveis, o que foi realizado utilizando a AFE. Esta, por meio dos indicadores "carga fatorial", "comunalidade", "percentagem da variância explicada", KMO e coeficiente de alfa de Cronbach (coeficiente de confiabilidade), indicara que as dimensões apresentadas poderiam ser refletidas nas variáveis propostas. Todos os constructos explicitados no modelo de mensuração apresentaram consistências internas, já que obtiveram valores superiores a 0,70 no critério do indicador "confiabilidade composta" e maiores que 0,5 para o indicador "variância extraída". Dessa forma, evidencia-se a confiabilidade do modelo que, ao final, engloba todas as variáveis latentes propostas no estudo, em sua composição estrutural.

A partir dessas variáveis, foi possível desenvolver um modelo estrutural de relacionamento para investigar o comportamento ambiental de residentes em destinos turísticos costeiros. Assim, observou-se a existência de correlação moderada entre as dimensões independentes utilizadas e a dimensão dependente "intenção pró-ambiental" $\left(\mathrm{R}^{2}: 0,40\right)$ e alta correlação entre as dimensões independentes utilizadas e a variável dependente "comportamento pró-ambiental" $\left(\mathrm{R}^{2}: 0,73\right)$. Notou-se fraca correlação entre a dimensão "apego ao lugar" e "intenção pró-ambiental", bem como a existência de relação não significativa entre a dimensão "apego ao lugar" e "comportamento pró-ambiental".

Dentro do horizonte de pesquisa utilizado, os resultados apontam que as variáveis de caráter cognitivo têm maior poder explicativo da intenção e do comportamento ambiental de residentes no destino analisado do que variáveis de caráter afetivo. Devido ao emprego de amostragem não probabilística, os resultados são conclusivos apenas para o público utilizado, indicando uma tendência de relacionamento de variáveis para outras situações. Eles podem auxiliar em futuras investigações acerca do comportamento pró-ambiental de residentes em áreas turísticas costeiras.

\section{REFERÊNCIAS}

Ajzen, I. (1991). The Theory of Planned Behavior. Organizational behavior and human decision processes, 50(2), 197-211.

Araújo, J. L. O., Araújo, A. C., \& A. C. Araújo (2010). Percepção ambiental dos residentes do Bairro Presidente Médici em Campina Grande-PB, no tocante à arborização local. REVSBAU, 5(2),67-81. 
Bonaiuto, M., Carrus, G., Martorella, H., \& Bonnes, M. (2002). Local identity processes and environmental attitudes in land use changes: the case of natural protected areas. Journal of Economic Psychology, 23(5), 631-653.

Bonnes, M., \& Bonaiuto, M. (2002). Environmental psychology: From spatial-physical environment to sustainable development. In R. B. Bechtel \& A. Churchman (Eds.), Handbook of environmental psychology (pp. 28-54). New York, NY: Wiley.

Buijs, A. E. (2009). Public support for river restoration: a mixed-method study into local residents' support for and framing of river management and ecological restoration in the Dutch floodplains. Journal of Environmental Management, 90(8), 2680-2689.

Carvalho, T. L. G. (2016). 0 turismo no Morro da Babilônia: do reflorestamento ao Ecoturismo. Revista Brasileira de Ecoturismo, 9(1), 11-28.

Caska, B. A. (1998). The search for employment: motivation to engage in coping behavior. Journal of Applied Social Psychology, 28(3), 206-224.

Corral-Verdugo, V., \& Pinheiro, J. Q. (1999). Condições para o estudo do comportamento pró-ambiental. Estudos de Psicologia, 4(1), 7-22.

Corrar, L. J., Paulo, E., \& Dias, J. M. F. (2009). Análise multivariada: para os cursos de administração, ciências contábeis e economia. São Paulo: Atlas.

Davies, J., Foxall, G. R., \& Pollister, J. (2002). Beyond the intention-behavior mythology: an integrated model of recycling. Marketing Theory, 2(1), 29-113.

Deboni, T. L., Mombach, G. N. N., Lopes, M. N., \& Simioni, F. J. (2015). Percepção e consciência ambiental: um estudo exploratório em Lages-SC. Geoambiente On-Line, 24, 97-113.

Duim, R. V. D., \& Caldeers, J. (2002). Biodiversity and tourism: impacts and interventions. Annals of Tourism Research, 29(3), 743-761.

Dunn, K. I., Mohr, P., Wilson, C. J., \& Wittert. G. A. (2011). Determinants of fast-food consumption: an application of the theory of planned behavior. Appetite, 57(2), 349-357.

Fandé, M. B., \& Pereira, V. F. G. V. (2014). Impactos ambientais do turismo: um estudo sobre a percepção de moradores e turistas no Município de Paraty-RJ. REGET, 18(3), 1170-1178.

Felippe, M. L., \& Kuhnen, A. (2012). 0 apego ao lugar no contexto dos estudos pessoasambiente: Práticas de pesquisa. Estudos de Psicologia, 29(4), 609-617.

Haden, S. S. P., Oyler, J. D., \& Humphreys, J. H. (2009). Historical, practical, and theoretical perspectives on green management: an exploratory analysis. Management Decision, 47(7), 1041-1055.

Hair Junior, J. F., Anderson, R. E., Tatham, R. L., \& Black, W. C. (2009). Análise multivariada de dados (6a ed.). Porto Alegre: Bookman

Huang, J. T. (2011). Application of planned behavior theory to account for college students' occupational intentions in contingent employment. Global Visions, 59(5), 455-466.

Hwang S. N., Lee C., Chen H.J. (2005). The relationship among tourists' involvement, place attachment and interpretation satisfaction in Taiwan's national parks. Tourism Management, 26(2), 143-156

Instituto Brasileiro de Geografia e Estatística. (2017). Rio de Janeiro, RJ: IBGE. Recuperado de https://bit.ly/2IJkwne

Karp, D. G. (1996). Values and their effect on pro-environmental behavior. Environment and Behavior, 28(1), 111- 133.

Keene, M., \& Pullin, A. S. (2011). Realizing an effectiveness revolution in environmental management. Journal of Environmental Management, 92(9), 2130-2135. 
Lamano-Ferreira, A.P. N., Morizono, R. C. F. C., Ribeiro, A. P., Kniess, C. T., \& Ramos, H. R. (2015). Dimensões do comportamento ecológico identificadas em moradores da cidade de São Paulo/SP. In XII Congresso de Ecologia do Brasil. São Lourenço, MG: Universidade Federal de Viçosa.

Le, Y., Hollenhorst, S., Harris, C., McLaughlin, W., \& Shook, S. (2006). Environmental management: a study of vietnamese hotels. Annals of Tourism Research, 33(2), 545-567.

Lee, H. T. (2013). Influence analysis of community resident support for sustainable tourism development. Tourism Management, 34, 37-46.

Lima, D. M. A., \& Bomfim, Z. A. C. (2009). Vinculação afetiva pessoa-ambiente: diálogos na psicologia comunitária e psicologia ambiental. Psico, 40(4), 491-497.

Luyet V., Schlaepfer R., Parlange, M.C., \& Buttler, A. (2012). A framework to implement stakeholder participation in environmental projects. Journal of Environmental Management, 111, 213-219.

Maloney, M. P., \& Ward, M. P. (1973). Ecology: let's hear it from the people - an objective scale for measurement of ecological attitudes and knowledge. American Psychologist, 28, 583-586.

Marôco, J. (2010). Análise de equações estruturais: fundamentos teóricos, software e aplicações. Pero Pinheiro: Report Number.

Moura, L. R. C., Veiga, R. T., Cunha, N. R. S., \& Moura, L. E. L. (2012). A teoria do comportamento planejado e sua modificação com a inclusão do comportamento passado: um estudo sobre o comportamento de tentar perder peso. Perspectiva, 36(136), 167-179.

Moura-Fé, M. M., \& Pinheiro, M. V. A. (2013). Os parques eólicos na zona costeira do Ceará e os impactos ambientais associados. Revista GeoNorte, 9(1), 22-41.

Ministério do Turismo. (2016). Turismo de sol e Praia movimenta o verão de 2014. Brasília, DF: Autor. Recuperado de https://bit.ly/2vjx9gf.

Oliveira, M. R. L., \& Nicolodi, J. L. (2012). A gestão costeira no Brasil e os dez anos do projeto Orla.: Uma análise sob a ótica do poder Público. Revista da Gestão Costeira Integrada, 12(1), 89-98.

Pol, E. (2003). A gestão ambiental, novo desafio para a psicologia do desenvolvimento sustentável. Estudos de Psicologia, 8(2), 235-243.

Richmond, L., Middleton, B. R., Gilmer, R., Grossman, Z., Janis, T., Lucero, S., Morgan, T., \& Watson, A. (2013). Indigenous studies speaks to environmental management. Environmental Management, 52(5), 1041-1045.

Santos, D. O., Veiga, R. T., \& Moura, L. R. C. (2010). Teoria do comportamento planejado decomposto. Organizações em contexto, 6(12), 78-106.

Sharifirad, G., Yarmohammadi, P., Azadbakht, L., Morowatisharifabad, M. A., \& Hassanzadeh, A. (2013). Determinants of fast food consumption among Iranian high school students based on planned behavior theory. Journal of Obesity, 2013, 1-7.

Smith, R. A. (1992). Beach Resort evolution: Implications for Planning. Annals of Tourism Research, 19(2), 304-322.

Straub, D. W., \& Watson, R. T. (2001). Research commentary: transformational issues in research IS and net-enabled organizations. Information Systems Research, 12(4), 337-345.

Tang, M., Fouad, N. A., \& Smith, P. L. (1999). Asian Americans' career choices: A path model to examine factors influencing their career choices. Journal of Vocational Behavior, 54(1), 142-157.

The Worldwatch Institute. (2013). O estado do mundo : a sustentabilidade ainda é possível? 2013. Salvador, BA. Recuperado de https://bit.ly/2vjx9gf. 
Van Hooft, E. A. J., \& De Jong, M. (2009). Predicting job seeking for temporary employment using the theory of planned behavior: the moderating role of individualism and collectivism. Journal of Occupational and Organizational Psychology, 82(2), 295-316.

Vasconcelos, F. P., \& Coriolano, L. N. (2008). Impactos socioambientais no litoral: um foco no turismo e na Gestão Integrada da Zona Costeira no estado do Ceará/Brasil. Revista de Gestão Costeira Integrada, 8(2), 259-275.

Walker, A. J., \& Ryan, R. L. (2008). Place attachment and landscape preservation in rural New England: a Maine case study. Landscape and Urban Planning, 86(2), 141-152.

Wanberg, C. R., Glomb, T. M., Song, Z., \& Sorenson, S. (2005). Job-search persistence during unemployment: a 10-wave longitudinal study. Journal of Applied Psychology, 90(3), 411-430.

Yuksel, A., Yuksel, F., \& Bilim, Y. (2010). Destination attachment: Effects on customer satisfaction and cognitive, affective and conative loyalty. Tourism Management, 31(2), 274-284.

Recebido em: $15 / 09 / 2017$

Aprovado em: 25/04/2018

\section{CONTRIBUIÇÕES}

Amanda Mirely Cipriano Soares: Definição do problema de pesquisa e objetivos, desenvolvimento da proposição teórica; realização da revisão bibliográfica e fundamentação teórica; escolha dos procedimentos metodológicos; coleta de dados; análise de dados; elaboração de tabelas, gráficos e figuras; realização de cálculos e projeções; revisão crítica do manuscrito; redação do manuscrito; adequação do manuscrito às normas da RTA.

Sérgio Marques Júnior: Definição do problema de pesquisa e objetivos; desenvolvimento da proposição teórica; realização da revisão bibliográfica e fundamentação teórica; escolha dos procedimentos metodológicos; coleta de dados; análise de dados; elaboração de tabelas, gráficos e figuras; realização de cálculos e projeções; revisão crítica do manuscrito; redação do manuscrito; adequação do manuscrito às normas da RTA.

Márcio Marreiro das Chagas: Definição do problema de pesquisa e objetivos; desenvolvimento da proposição teórica; escolha dos procedimentos metodológicos; análise de dados; elaboração de tabelas, gráficos e figuras; revisão crítica do manuscrito; redação do manuscrito; adequação do manuscrito às normas da RTA. 